\title{
Perspective study of exotics and flavour baryons in antiproton-proton annihilation and proton-proton collisions
}

\author{
Mikhail Barabanov, Alexander Vodopyanov \\ Joint Institute for Nuclear Research, 141980 Joliot-Curie 6 Dubna, Russia
}

\begin{abstract}
The spectroscopy of exotic states with hidden charm is discussed. Together with charmonium, these provide a good tool for testing theories of the strong interactions including both perturbative and non-perturbative QCD, lattice QCD, potential and other phenomenological models. An elaborated analysis of exotics spectrum is given, and attempts to interpret recent experimentally observed states with masses above the $D \bar{D}$ threshold region are considered. Experimental results from different collaborations (BES, BaBar, Belle, LHCb) are analyzed with special attention given to recently discovered hidden charm states. Some of these states can be interpreted as higher-lying charmonium states and others as tetraquarks with hidden charm. It has been shown that charged/neutral tetraquarks must have their neutral/charge partners with mass values differ by at most a few $\mathrm{MeV} / \mathrm{c}^{2}$, hypotheses that tend to coincide with those proposed by Maiani and Polosa. However, measurements of different decay modes are needed before firm conclusions can be made. These data can be derived directly from the experiments using ahigh quality antiproton beam with momentum up to $15 \mathrm{GeV} / \mathrm{c}$ and proton-proton collisions with momentum up to $26 \mathrm{GeV} / \mathrm{c}$.
\end{abstract}

\section{Introduction}

The study of strong interactions and hadron matter in the process of antiproton-proton annihilation and proton-proton collisions seems to be a challenge nowadays. One of the main goals of contemporary physics is to search for new exotic forms of matter, some of which will be manifest in the form of charmed hybrids and multiquark states, such as meson molecules and tetraquarks [1,2]. Studies of the spectra of charmed hybrids $c \bar{c} g$ and tetraquarks containing hidden charm $\left(c q \bar{c} \bar{q}^{\prime}, q\right.$ and $q^{\prime}=u, d$, $s$ ), together with completion of the charmonium spectrum are promising approaches for the development of an understanding of the dynamics of quark interactions at small distances. They provide a good testing tool that challenges theories of strong interactions: QCD in both perturbative and non-perturbative regimes, QCD inspired potential models, phenomenological models, and nonrelativistic QCD and LQCD.

Charmed hybrids $c \bar{c} g$ are hypothesized $c \bar{c}$ states with an excited gluonic degree of freedom. These states are described by different models and calculational schemes (LQCD, bag model, flux tube model) $[1,2]$. All model predictions and calculations tend to agree that the masses of the lowest-lying charmonium hybrids are between 3.9 and $4.6 \mathrm{GeV} / \mathrm{c}^{2}$ and that the state with $J^{P C}=1^{-+}$has the lowest mass. Until now, discussions have been focused only around the lowest-lying charmonium hybrids. 
Four of these states $J^{P C}=2^{-+}, 1^{-+}, 1^{--}, 0^{-+}$correspond to a $c \bar{c}$ pair with $J^{P C}=0^{-+}$or $1^{--}$, coupled to a gluon in the lightest mode with $J^{P C}=1^{+-}$. Four other states with a $c \bar{c}$ pair with $J^{P C}=2^{+-}, 1^{+-}, 1^{++}, 0^{+-}$ coupled with a $J^{P C}=1^{-+}$mode are, probably, a bit heavier. The expected mass splitting between the $1^{-}$ ${ }^{+}$and $0^{+-}$states is about $150-250 \mathrm{MeV} / \mathrm{c}^{2}$. Three of these eight low-lying charmonium hybrids have spin-exotic quantum numbers $1^{-+}, 0^{+-}, 2^{+}$, and for these mixing effects with nearby $c \bar{c}$ states are excluded, making their experimental identification especially unambiguous. The next possible hybrid states with quantum numbers $2^{++}, 2^{+-}, 1^{++}, 1^{+-}, 0^{+-}, 0^{++}$correspond to $c \bar{c}$ pair with quantum numbers $J^{P C}=1^{+-}$or $J^{P C}=(0,1,2)^{++}$coupled to a gluon that, in the lightest mode, has $J^{P C}=1^{+-}$. The states with quantum numbers $2^{--}, 2^{-+}, 1^{--}, 1^{-+}, 0^{-+}, 0^{--}$correspond to $c \bar{c}$ pair with quantum numbers $J^{P C}=1^{+-}$or $J^{P C}=(0,1,2)^{++}$that are coupled to a gluon mode with $J^{P C}=1^{-+}$. One can find a possibility of the existence of hybrid state with the exotic quantum numbers $J^{P C}=0^{--}$. The most interesting and promising decay channels for charmed hybrids are as follows: $\bar{p} p \rightarrow \widetilde{\eta}_{c 0,1,2}\left(0^{-+}, 1^{-+}, 2^{-+}\right) \eta \rightarrow \chi_{c 0,1,2}(\eta$, $\pi \pi ; \ldots) ; \bar{p} p \rightarrow \widetilde{h}_{c 0,1,2}\left(0^{+-}, 1^{+-}, 2^{+-}\right) \eta \rightarrow \chi_{c 0,1,2}(\eta, \pi \pi ; \ldots) ; \bar{p} p \rightarrow \widetilde{\Psi}\left(1^{--}, 2^{-}\right) \rightarrow J / \Psi(\eta, \omega, \pi \pi, \ldots) ; \bar{p} p \rightarrow$ $\widetilde{\eta}_{c 0,1,2}, \widetilde{h}_{c 0,1,2}, \tilde{\chi}_{c 1}\left(0^{-+}, 1^{-+}, 2^{-+}, 0^{+-}, 1^{+-}, 2^{+-}, 1^{++}, 2^{++}\right) \eta \rightarrow D \bar{D}_{J}^{*} \eta$

In the last few years we have witnessed the discovery of a number of narrow hadron resonances with hidden charm that do not match the standard quark-antiquark interpretation, and have, thereby, been named exotic hadrons [2 -5]. This has called for alternative interpretations of their inner structure. One of the possible explanations is that these particles are loosely bound molecules of open charm mesons. Another possibility is that new aggregation patterns of quarks in matter are possible. We follow the suggestion of using diquarks as building blocks. Light diquarks have been an object of several lattice studies. The idea that the coloured diquark can be handled as a constituent building block is at the core of this approach. Two generic types of multiquark states have been described in the literature [3 - 5]. The first one, the molecular state, is comprised of two charmed mesons bound together to form a molecule. These states are, by nature, loosely bound. Molecular states bound through two mechanisms: quark/colour exchange interactions at short distances and pion exchange at a large distance. Since the mesons inside the molecule are weakly bound, they tend to decay as if they are free. The second type is a tightly bound four-quark state, a so-called tetraquark that is predicted to have properties that are distinct from those of a molecular state. In the model of Maiani [6], for example, the tetraquark is described as a diquark-diantiquark structure in which the quarks group into the colour-triplet scalar and vector clusters with interactions that are dominated by a simple spin-spin interaction. Here, strong decays are expected to proceed via rearrangement processes followed by dissociation that gives rise, for example, to processes such as: $\bar{p} p \rightarrow X \rightarrow J / \Psi \rho \rightarrow J / \Psi \pi \pi ; \bar{p} p \rightarrow X \rightarrow$ $J / \Psi \omega \rightarrow J / \Psi \pi \pi \pi, \quad \bar{p} p \rightarrow X \rightarrow \chi_{c J} \pi ; \bar{p} p \rightarrow X \rightarrow D \bar{D}^{*} \rightarrow D \bar{D} \gamma ; \bar{p} p \rightarrow X \rightarrow D \bar{D}^{*} \rightarrow D \bar{D} \eta$. A prediction that distinguishes tetraquarks containing a $c \bar{c}$ pair from conventional charmonia is possible existence of multiplets that include members with non-zero charge $c u \bar{c} \bar{d}$, strangeness $c d \bar{c} \bar{s}$, or both $c u \bar{c} \bar{s}$.

\section{The model of confinement for resonance decay products}

The actual calculation of the dynamics of a resonance is an impracticable task, but we considered the particular moment when two decay products are out of range for a mutual interaction and no potential energy is involved in calculations. This happens at a distance $R$ for a given group of resonances, the distance at which the resonance ceases to exist and two or more decay products are only the objects to consider. At that moment there is no translational energy of the decay products and only the orbital kinetic energy is relevant for each of them. The spin rotational energy is described with the relativistic spherical symmetric top model with quantized kinetic energy. So the resonance decay products are described as a collection of two tops in the same rotational state. Therefore the decay channels may be joint to the groups with the properties of quantum objects. Inside each group the decay products momenta are quantized relatively characteristic length $R$. Thus we can consider the resonances as the 
states of relativistic quantum system. It was shown that the dynamic of the system is derived from SU(2)group generators [7, 8]. Hence SU(2)-group plays the fundamental role in quantization of energymomentum.

Let us define the set of generators of $S O(4)$ group

$$
\vec{M}=[\vec{r} \times \vec{p}], \vec{N}=R \vec{p}+\vec{r}(\vec{r} \vec{p}) / R,
$$

The linear combinations of these orthonormal operators

$$
\mu_{ \pm}=(\vec{M} \pm \vec{N})
$$

contribute two sets of generators of the $\mathrm{SU}(2)$ group. Thus the $S U(2)$ group generates the action on a three-dimensional sphere $S^{3}$.

This action consists of the translation with whirling around the direction of translation.

We get:

$$
H=\frac{1}{2 m R^{2}}\left\{2 \hbar+\left(\vec{\mu}_{ \pm}, \vec{\sigma}\right)\right\}\left\{2 \hbar+\left(\vec{\mu}_{ \pm}, \vec{\sigma}\right)\right\},
$$

where $\vec{\sigma}$ is the spin operator, $\hbar$ is the Plank constant, $m$ is the top mass.

The spectrum is:

$$
H \Psi_{n}=\frac{\hbar^{2}}{2 m R^{2}}(n+1)^{2} \Psi_{n}, n=0,1,2 \ldots,
$$

The wave function $\Psi_{n}=\left|L S J M_{J}\right\rangle$ was taken as eigenfunction of whole momentum $J^{2}=\left(\mu^{ \pm}+s^{2}\right)$ of the top.

Let us generalize this concept to the relativistic case:

$$
M(R)=\sqrt{m_{a}^{2}+\frac{1}{R^{2}}\left(\left(\vec{\sigma} \vec{\mu}_{ \pm}\right)+2 \hbar\right)^{2}}+\sqrt{m_{b}^{2}+\frac{1}{R^{2}}\left(\left(\vec{\sigma} \vec{\mu}_{ \pm}\right)+2 \hbar\right)^{2}},
$$

where $m_{a}$ and $m_{b}$ are the masses of resonance decay products.

The spectrum is:

$$
E=\sqrt{m_{a}^{2}+\frac{\hbar^{2}(n+1)^{2}}{R^{2}}}+\sqrt{m_{b}^{2}+\frac{\hbar^{2}(n+1)^{2}}{R^{2}}}, n=0,1,2 \ldots,
$$

Finally, the formula for resonance mass spectrum has the form:

$$
E=\sqrt{m_{a}^{2}+P_{n}^{2}}+\sqrt{m_{b}^{2}+P_{n}^{2}}=\sqrt{m_{a}^{2}+\left[n * P_{0}\right]^{2}}+\sqrt{m_{b}^{2}+\left[n * P_{0}\right]^{2}}
$$

where $R \rightarrow a+b$ - a binary decay channel (we use the system where $\hbar=c=1$ ), $m_{a}, m_{b}$ - the masses of resonance binary decay products, $P_{0}$ - basic momentum, $P_{n}$ - asymptotic momentum of their relative motion.

\section{Calculation the exotics spectrum}

For these purposes we have performed an elaborated analysis of the spectrum of charmed hybrids and tetraquarks with the hidden charm with masses above the $D \bar{D}$ threshold region. An analysis of spectrum of charmonium states was carried out earlier [9, 10]. In particular, different decay modes of charmed hybrids and tetraquarks, such as decays into charmonium and light mesons and decays into $D \bar{D}_{J}{ }^{*}$ and $D \bar{D}^{*}$ pairs, were analyzed. Special attention was given to recently discovered states with 
hidden charm [2 - 5]. Experimental results from different collaborations like Belle, BaBar, LHCb, BES were used. It was demonstrated $[11,12]$ that the dynamics of the decaying resonant system (charmonium, charmed hybrid and tetraquark) is determined by a set of $S U(2)$ group generators. Mathematical aspects of spin particle motion on the spherical surface $S^{3}$ defined in four-dimensional Euclidean space were considered [7, 8]. The radius of the sphere is a constant. Conformity with the three-dimensional actual space is achieved by transition to the spherical surface $S^{3}$. The authors shown $[11,12]$ that two manifolds of $S U(2)$ group generators specify the equation of motion of particles with spin $1 / 2$ on the tree-dimensional sphere $S^{3}$ in four-dimensional Euclidean space. Subsequently, they generalized the aforesaid to particles with spin equals 1 . In this confinement model, a geometrical point, lying on the sphere, is associated with relativistic spherically symmetric top. The totality of resonant states with identical quantum numbers is interpreted as radially excited states of the same quantum system (in our case charmonium, charmed hybrids or tetraquark). The proposed model was developed in this paper. According to a combined approach based on the quarkonium potential model and a confinement model $[11,12]$, more than twenty charmed hybrids are expected to exist in the mass region under consideration (see Figs. 1,2). Charmed hybrids with exotic quantum numbers are marked with dark shading and charmed hybrids with nonexotic quantum numbers are indicated with light shading. The results of calculations for the hybrids are in good agreement with the well accepted picture that the quartet $1^{--},(0,1,2)^{-+}$is lower in mass than $1^{++},(0,1,2)^{+-}$. The mass splitting between the states $1^{-+}$and $0^{+-}$is about $200 \mathrm{MeV} / \mathrm{c}^{2}$. More than twenty tetraquarks with hidden charm (see Fig. 3) are expected to exist in the mass region above the $D \bar{D}$ threshold. The black-white boxes correspond to the recently revealed $X Y Z$ states with the hidden charm that may be interpreted as tetraquarks. White boxes correspond to the tetraquark states that have yet to be found. The possible existence of these states is predicted in the framework of the combined approach. It has been shown that charge/neutral tetraquarks with hidden charm must have neutral/charged partners with mass values that differ at most by a few $\mathrm{MeV} / \mathrm{c}^{2}$. This assumption coincides with that proposed earlier by Maiani and Polosa [13] and can shed light on the nature of neutral $X(3872), \mathrm{X}(4350)$ and charged $Z_{\mathrm{c}}(3885)^{ \pm}, Z_{\mathrm{c}}(3900)^{ \pm}, Z_{\mathrm{c}}(4020)^{ \pm}, Z_{\mathrm{c}}(4025)^{ \pm}, Z_{\mathrm{c}}(4200)^{ \pm}, Z_{\mathrm{c}}(4050)^{ \pm}, Z_{c}(4250)^{ \pm} Z_{\mathrm{c}}(4430)^{ \pm}$ states. The quantum numbers $J^{P C}$ of the $X(3872)$ meson have been recently determined by LHCb [14]. We find that the $X(3872)$ can be interpreted as tetraquark state with $J^{P C}=1^{++}$, and the $X(4350)$ as the tetraquark state with $J^{P C}=2^{++}$. The charged $\mathrm{Z}_{\mathrm{c}}(3900)^{ \pm}$state that was recently observed by BES $[15,16]$ and Belle [17], the $Z_{c}(4020)^{ \pm}$seen by BES $[18,19]$, the $Z_{c}(4050)^{ \pm}$and $Z_{c}(4250)^{ \pm}$seen by Belle decaying to $\chi_{c l} \pi^{ \pm}[20]$ and the $Z_{c}(4430)^{ \pm}$charged state seen by Belle decaying to $\Psi^{\prime} \pi^{ \pm}$[21] can be identified as charge tetraquarks with $J^{P C}=1^{+}$. (The proposed approach doesn't distinguish the states $Z_{c}(3900)^{ \pm}[15,17]$ and $Z_{c}(3885)^{ \pm}[16]$ as well the $Z_{c}(4020)^{ \pm}[18]$ and $Z_{c}(4025)^{ \pm}$[19] states.) The values of their masses and widths coincide in the framework of the combined approach. Two states (one charged and one neutral) with $J^{P C}=1^{++}$are expected to exist in the mass range of $4200-4300 \mathrm{MeV} / \mathrm{c}^{2}$. The $Z_{c}(4200)^{ \pm}$charged state recently reported by Belle [22] may be a good candidate for the one of them. 


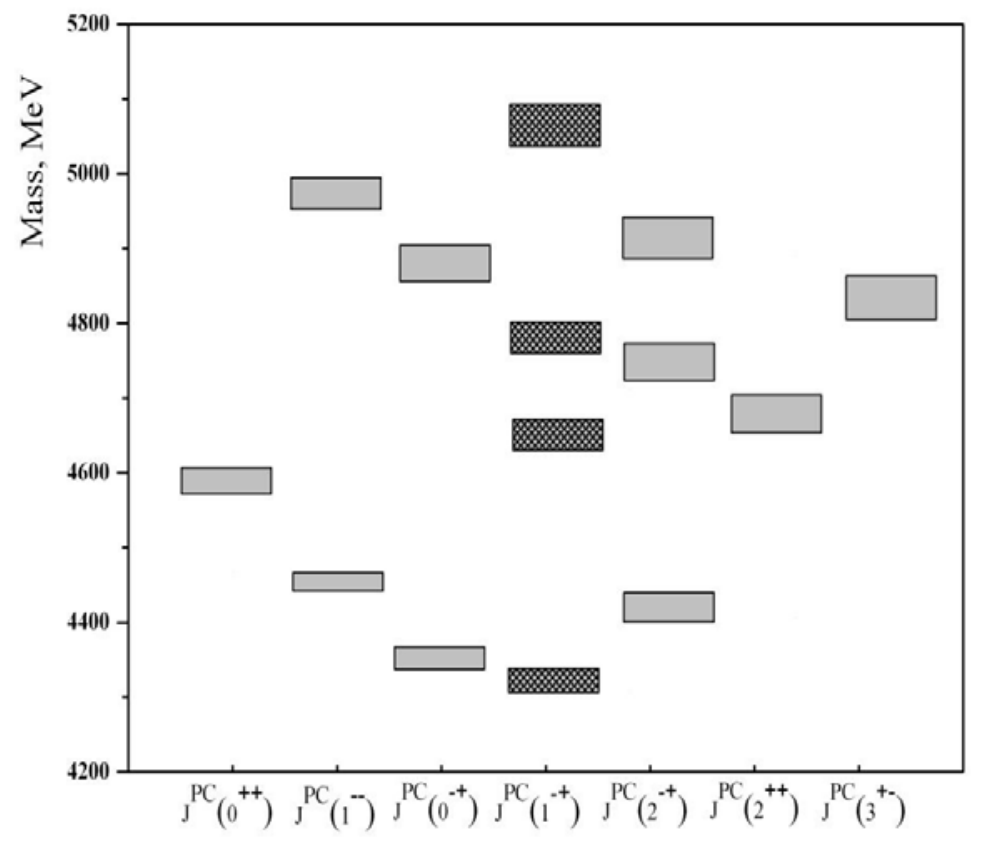

Figure 1. The spectrum of charmed hybrids with quantum numbers $J^{P C}=3^{+-}, 2^{++}, 2^{-+}, 1^{-+}, 1^{--}, 0^{-+}, 0^{++}$. Charmed hybrids with exotic quantum numbers are marked with dark shading and charmed hybrids with nonexotic quantum numbers are indicated with light shading.

All these states are predictions in the combined approach.

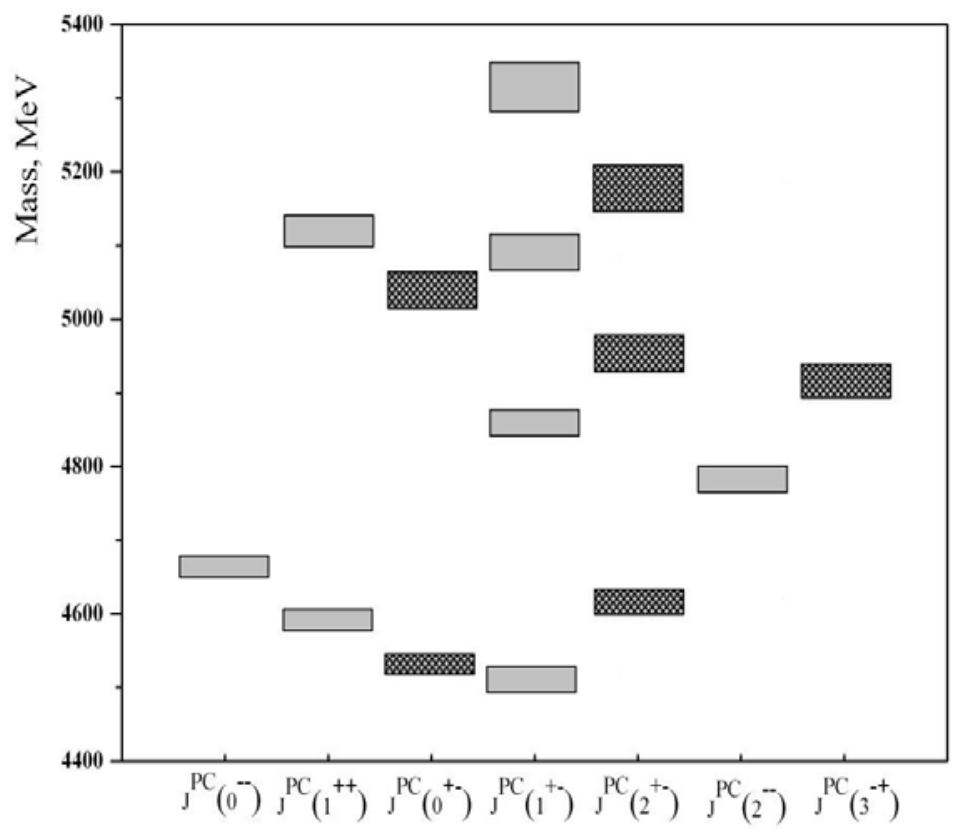

Figure 2. The spectrum of charmed hybrids with quantum numbers $J^{P C}=3^{-+}, 2^{--}, 2^{+-}, 1^{+-}, 1^{++}, 0^{+-}, 0^{--}$.

Charmed hybrids with exotic quantum numbers are marked with dark shading and charmed hybrids with nonexotic quantum numbers are indicated with light shading.

All these states are predictions in the combined approach. 


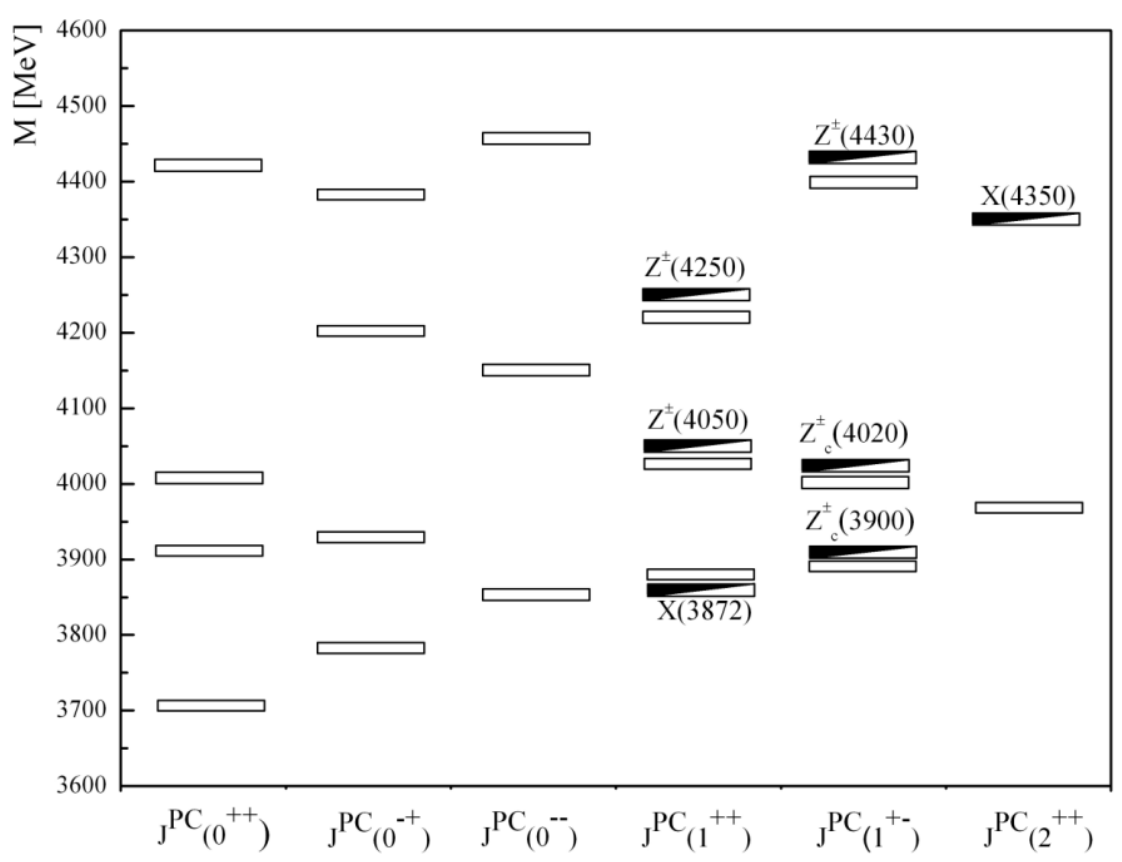

Figure 3. The spectrum of tetraquarks with hidden charm. The black-white boxes correspond to the recently revealed $X Y Z$ states with the hidden charm that may be interpreted as tetraquarks. White boxes correspond to the tetraquark states that have yetto be found. The existence of these states is predicted in the framework of the combined approach.

To confirm that the predicted states actually exist and can be found experimentally, their widths and branching ratios were calculated $[10,12]$. The feature of the considered states is their narrowness compared with light unflavored mesons, baryons and hybrids. The states we find in this model have small widths; their values are of the order of several tens of $\mathrm{MeV}$. This fact facilitates experimental searches. The values of the calculated widths coincide (within the experimental error) with the experimentally determined values for the $X Y Z$ particles; the correspondence of the mass values has been discussed above. This fact strongly suggests that some of the $X Y Z$ particles may be interpreted as higher-lying charmonium states $[9,10]$ and tetraquarks as it can be verified by the experiments with antiproton beams with momentum up to $15 \mathrm{GeV} / \mathrm{c}$ and proton-proton collisions with momentum up to $26 \mathrm{GeV} / \mathrm{c}$. The values of branching ratios in the considered decay channels of charmonium and exotics are of the order of $10^{-1}-10^{-2}$ dependent of their decay mode. From this one can conclude that the branching ratios are significant and searches for charmonium and exotics, and studies of the main characteristics of their spectrum seem to be promising.

\section{Conclusion}

The prospects for future exotics research are related with the results obtained below:

A combined approach has been employed to study charmonium and exotics on the basis of the quarkonium potential model and a confinement model that uses a three-dimensional sphere embedded into the four-dimensional Euclidian space of the decay products.

The most interesting and promising decay channels of charmed hybrids and tetraqurks with the hidden charm have been analyzed. Many new states above $D \bar{D}$ threshold are expected to exist in the framework of this model.

The recently discovered states with the hidden charm above the $D \bar{D}$ threshold ( $X Y Z$ particles) have been analyzed. Ten of these states can be interpreted as higher-lying tetraquark states with hidden 
charm. The necessity of further studies of the $X Y Z$ particles and improved measurements of their main characteristics has been demonstrated.

\section{References}

1. W. Erni et al., arXiv:0903.3905v1 [hep-ex] (2009)

2. N. Brambilla et al., Eur. Phys. J. C 71: 1534, 1 (2011)

3. J. Beringer et al., Reviewof Particle Physics, Phys. Rev. 86 (2012)

4. S. Godfrey and S. Olsen, Ann. Rev. Nucl. Part. Sci. 58, 51 (2008)

5. S. Olsen, arXiv : 0909.2713v1 [hep-ex] (2009)

6. L. Maiani, F. Piccinini, A.D. Polosa, V. Riquer, Phys. Rev. D 71, 014028 (2005)

7. J. Keller, R. Yamaleev, A. Rodrigues, Adv. in Appl. Clifford Algebras 8, N.2, 235 (1998)

8. F. Gareev, J. Keller, R. Yamaleev, Adv. in Appl. Clifford Algebras 8, N.2, 255 (1998)

9. M.Yu. Barabanov, A.S. Vodopyanov, Fizika Elementarnyh Chastits Atomnogo Yadra Pisma, 8, N.10, 63 (2011) / Phys. Part. Nucl. Lett. 8, N.10, 1069 (2011).

10. M.Yu. Barabanov, A.S. Vodopyanov, S. L. Olsen, YadernayaFizica, 77, N.1, 1 (2014) / Phys. At. Nucl., 77, N.1,1 26 (2014).

11. M.Yu. Barabanov et al., Russ. Phys. J., 50, 1243 (2007)

12. M.Yu. Barabanov et al., Hadronic J., 32, N.2, 159 (2009)

13. L. Maiani, F. Piccinini, A.D. Polosa, V. Riquer, Phys. Rev. Lett. 99, 182003 (2007)

14. R. Aaij et al. (LHCb Collaboration), Phys. Rev. Lett. 110, 222001 (2013)

15. M. Ablikim et al. (BESIII Collaboration), Phys. Rev. Lett. 110, 252001 (2013)

16. M. Ablikim et al. (BESIII Collaboration), Phys. Rev. Lett. 112, 022001 (2014)

17. Z.Q. Liuet al. (Belle Collaboration), Phys. Rev. Lett. 110, 252002 (2013)

18. M. Ablikim et al. (BESIII Collaboration), Phys. Rev. Lett. 111, 242001 (2013)

19. M. Ablikim et al. (BESIII Collaboration), Phys. Rev. Lett. 112, 132001 (2014)

20. R. Mizuk et al. (Belle Collaboration), Phys. Rev. D 78, 072004 (2008)

21. S.-K. Choiet al. (Belle Collaboration), Phys. Rev. Lett. 100, 142001 (2008)

22. K. Chilikinet al. (Belle Collaboration), arXiv: 1408.6457v3 [hep-ex] (2014) 